\title{
A Robust Algorithm for Classification and Diagnosis of Brain Disease Using Local Linear Approximation and Generalized Autoregressive Conditional Heteroscedasticity Model
}

\author{
Ali Hamzenejad ${ }^{1}$, Saeid Jafarzadeh Ghoushchi ${ }^{2}$, Vahid Baradaran ${ }^{1}(\mathbb{D})$ and Abbas Mardani ${ }^{3,4, *(1)}$ \\ 1 Department of Industrial engineering, Islamic Azad University Tehran North Branch, Tehran 1477893855, \\ Iran; ali.hamzenejad@gmail.com (A.H.); v_baradaran@iau-tnb.ac.ir (V.B.) \\ 2 Department of Industrial engineering, Urmia University of Technology, Urmia 5716693188, Iran; \\ s.jJafarzadeh@uut.ac.ir \\ 3 Informetrics Research Group, Ton Duc Thang University, Ho Chi Minh City 758307, Vietnam \\ 4 Faculty of Business Administration, Ton Duc Thang University, Ho Chi Minh City 758307, Vietnam \\ * Correspondence: abbas.mardani@tdtu.edu.vn
}

Received: 5 July 2020; Accepted: 24 July 2020; Published: 2 August 2020

\begin{abstract}
Regions detection has an influence on the better treatment of brain tumors. Existing algorithms in the early detection of tumors are difficult to diagnose reliably. In this paper, we introduced a new robust algorithm using three methods for the classification of brain disease. The first method is Wavelet-Generalized Autoregressive Conditional Heteroscedasticity-K-Nearest Neighbor (W-GARCH-KNN). The Two-Dimensional Discrete Wavelet (2D-DWT) is utilized as the input images. The sub-banded wavelet coefficients are modeled using the GARCH model. The features of the GARCH model are considered as the main property vector. The second method is the Developed Wavelet-GARCH-KNN (D-WGK), which solves the incompatibility of the WGK method for the use of a low pass sub-band. The third method is the Wavelet Local Linear Approximation (LLA)-KNN, which we used for modeling the wavelet sub-bands. The extracted features were applied separately to determine the normal image or brain tumor based on classification methods. The classification was performed for the diagnosis of tumor types. The empirical results showed that the proposed algorithm obtained a high rate of classification and better practices than recently introduced algorithms while requiring a smaller number of classification features. According to the results, the Low-Low sub-bands are not adopted with the GARCH model; therefore, with the use of homomorphic filtering, this limitation is overcome. The results showed that the presented Local Linear (LL) method was better than the GARCH model for modeling wavelet sub-bands.
\end{abstract}

Keywords: Magnetic Resonance Imaging (MRI); wavelet transform; GARCH; LLA; LDA; KNN

\section{Introduction}

Electromagnetic imaging techniques provide valuable information about the human body. One of these methods is the Magnetic Resonance Imaging (MRI) of the brain [1]. One major area of research that has expanded in medical engineering involves diagnostic tools by machine control for a quicker and easier inference, which can be a great help for physicians in clinical medicine. Therefore, in recent years, mathematical methods have attracted much attention to the analysis of neural network data [2]. Brain images are considered as interesting subjects in the mathematical application and diagnosis of brain disorders in a patient [3]. The MRI can be used to examine the status of the brain tissue and discover whether or not there is a disease [4]. In MRI imaging, the patient is exposed to a strong 
magnetic field, after which radio waves are leaked toward him. The body's tissues emit another radio wave in response to this position. By receiving these radio waves emitted from the patient's body and by analyzing these waves via a powerful computer, images are created on the device monitor that show the levels of the target organ parts. The next step involves extracting features.

The Two-Dimensional Wavelet Transform (DWT) and the Principal Component Analysis (PCA) were the methods that were used to extract the features of the images [5,6]. Then, the classification methods were used to diagnosis the disease type in the brain [7,8]. Chaplot, et al. [9] used two-dimensional DWT sub-bands to extract the features in their research on Alzheimer's Disease (AD). Additionally, Daubechies filters were used as a filtering technique. The outcome illustrated that the Support Vector Machine (SVM) with radial base function and the polynomial kernel has a higher performance than linear neural networks and SVM [10,11]. Hackmack, et al. [12] used multidimensional complex wavelet transformations, and then linear SVM, to determine multi-scale brain images. The results showed that low-band scales include more information than high-frequency values. Maitra and Chatterjee [13] presented a Slantlet deformation-developed DWT-to extract the containing features of the brain's images. The Fuzzy C-Meaning (FCM) method has been used to analyze the brain MRI, based on the characteristics of the image histogram, in order to determine a healthy subject from Alzheimer's disease. Ramathilagam, et al. [14] used the c-means fuzzy modified algorithm to divide the brain MRI image with a T1-T2 weight. Since the c-means standard factor is intensively sensitive to the noise-induced area during extraction, the authors proposed to repeat the dist-max algorithm before executing the method.

Rivest-Hénault and Cheriet [15] used a local linear representation to model the brain tissue, after which regional models were embedded in the framework of the surface set in order to control the spatial integrity of division. Hussain, et al. [16] classified the images as normal or abnormal using (Back-Propagation Neural Networks) BPNN feed-forward, with characteristics derived from dynamic statistics and 2D-DWT. Bhattacharyya and Kim [17] presented an image segmentation technique for detecting a tumor with MRI images. The existing thresholding techniques produced different results in each image. Therefore, in order to achieve a satisfactory result in the brain tumor image, they presented a methodology that found the tumor to be unique. Kim, et al. [18] studied the diagnosis of Alzheimer's disease based on the Electroencephalogram (EEG) signal of the brain using genetic algorithms and neural networks. One of the remarkable points in this study was the ability to differentiate Alzheimer's patients from the mild stage of healthy subjects with $82 \%$ accuracy using a single-channel EEG signal [19]. Additionally, in another study, the comparison of the EEG signaling disorder of healthy people with that of brain tumor patients was calculated by entropy. According to the results, in low-frequency patients, low-rhythms of EEG signals such as Delta and Theta bands have a higher power spectrum than for healthy people. Gholipour, et al. [20] described the use of a completely new automated software algorithm using the standard MRI sequence before and after Contra T1. The T1 weighs in before and after Contra, and the images are automatically interconnected and normalized. The volume of tumor growth is automatically calculated. In their study, they were able to test a method for calculating the size of the tumor when it was enlarged by the collapse of the cavity and, of course, when the enlarged tumor was covered with semi-autogenous blood in a cutaneous cavity. It detected an increase in tumor volume among blood products, which rarely reduced measurements when using other techniques. Their approach seems to overcome many challenges by assessing the response to increased brain tumors and leading to more validation. Zacharaki, et al. [21] studied machine-learning algorithms that automatically identify the relevant features and are desirable for brain tumor differentiation. They studied various machine-learning techniques for classifying the brain tumor based on the features extracted from conventional MRI and perfusion. Their study was performed by mutual validation of Leave-One-Out (LOO) exodus on 101 brain tumors, obtained using a pack evaluation in combination with the best first-order algorithm and K-Nearest Neighbour (KNN) algorithm classification, reaching 96.9\%. When differentiated, it became Glimatic and 94.5\% when distinguished from a low-grade neoplasm. Fritzsche, et al. [22] completed a study of 15 patients 
with brain tumors and 18 patients with Mild Cognitive Impairment (MCI); eight remained stable in a three-year follow-up, and 15 were healthy individuals. The classification was also improved by limiting the analysis to the left-brain hemisphere. Devanand, et al. [23], using morphometric mapping of MRI, evaluated the local changes of the hippocampus grains and entorhinal cortex in predicting the transformation from a MCI cognitive impairment to an AD brain tumor. In the MCI model, Cox regression models for the conversion time to conversion converters were made for $A D(n=31)$ and 99 non-converted controls for age, sex, and education. In Zöllner, et al. [24], the performances of reduction features such as the Pearson correlation coefficient, principal components analysis, and independent component analysis in the classification of Glioma's disease were analyzed using a backup vector machine classifier.

Afshar, et al. [25] studied classification using CapsNets for the detection of brain tumors in order to present a developed architecture with higher accuracy. Their findings indicated that the presented method could overcome Convolutional Neural Networks (CNNs) successfully. Mohan and Subashini [26] provided a clinical study of brain tumor imaging related to gliomas. They used related methods of segmentation and classification. Huang, et al. [27] proposed an algorithm based on the rough set method. They presented a hybrid method with the use of FCM. Initially, the feature table was set based on FCM clustering amounts. Then, the relationship among features showed similarity criteria in each cluster.

In this paper, we presented three algorithms, named WGK, D-WGK, and WLK. The first presented method is Wavelet-GARCH-KNN (WGK). In this method, we first used a two-stage 2D-DWT to decompose the input images into sub-bands of wavelets. The reached wavelet coefficients were features of classification. Then, the GARCH model was used for feature extraction with the use of HH1, HL1, LH1, and second stage HH2, HL2, LH2. Because of the incompatibility of Local Linear (LL) with the GARCH model, this sub-band was ignored [28]. To reduce the number of features, the PCA and PCA + LDA method was then used with the extracted feature brain lesion being classified via KNN methods. The results are illustrated in the results section. The second presented method is named Developed Wavelet-GARCH-KNN (D-WGK). In the second method, we overcame the limitation of the WGK algorithm using homomorphic filtering before a wavelet transformation. Therefore, the LL2 sub-band participated in the GARCH model. Then, similarly to the WGK method, the KNN method was designated for the classification of brain tumors. The third method was Wavelet-LLA-KNN (WLK). In this method, all sub-bands of the wavelet decomposition were used for modeling with the LLA algorithm. The remaining part of the third method was also similar to the WGK and D-WGK method.

\section{Methods and Materials}

\subsection{Image Processing}

The modern world of today allows digital images to be analyzed and stored [29]. To get better results, it is sometimes necessary to make changes to these images. These changes have three main purposes: processing, analysis, and image perception. For this reason, computer image processing systems have been developed to perform these operations with better speed and accuracy. In these systems, four major processes occur pre-processing, image quality enhancement, image transformation, and classification and segmentation. In these methods, using mathematical science, rules have been created by computers to simulate human visual elements, and this is an aspect of image analysis that is used for specific purposes. Computer Vision is the analysis of scientific images in various scientific branches such as medicine, engineering, molecular imaging, astronautics, security, etc. Modern digital technology has made it possible to manipulate multidimensional signals from systems ranging from simple digital circuits to multiple parallel computers [30,31]. 


\subsection{Discrete Wavelet Transform (DWT)}

Let $f(x) \in L^{2}(\boldsymbol{R})$ be the function wavelet expansion related to the wavelet $\psi(x)$ and scaling $\varphi(x)$ function [20]; we then have:

$$
\begin{gathered}
f(x)=\frac{1}{\sqrt{M}} \sum_{k} W_{\varphi}\left(j_{0}, k\right) \phi_{j_{0}, k}(x)+\frac{1}{\sqrt{M}} \sum_{j=j_{0}}^{J} \sum_{k} W_{\psi}(j, k) \psi_{j, k}(x) \\
W_{\varphi}\left(j_{0}, k\right)=\frac{1}{\sqrt{M}} \sum_{x=0}^{M-1} f(x) \phi_{j 0, k}(x) \\
W_{\psi}(j, k)=\frac{1}{\sqrt{M}} \sum_{x=0}^{M-1} f(x) \psi_{j, k}(x) \quad j \geq j_{0} \\
\phi_{j, k}(x)=2^{\frac{j}{2}} \phi\left(2^{j} x-k\right) \\
\psi_{j, k}(x)=2^{\frac{j}{2}} \psi\left(2^{j} x-k\right)
\end{gathered}
$$

where $f(x)$ is the input variable as a vector, and $\varphi_{j_{0}, k}(x)$ and $\psi_{j, k}(x)$ are the scaling coefficient and wavelet coefficient, respectively. $x=0,1, \ldots, M-1, j=0,1, \ldots, J-1, k=0,1,2, \ldots, M-1$, where $M$ is the number of samples to be transformed that is equal to $2^{J}, J$ is the number of transformation levels, and $j_{0}$ is a random starting scale. The expansion function is a series of crisp numbers; it is also called the discrete wavelet transform of $f(x)$. The representation of the discrete function of $f(x)$ can be written as a weighted summation of wavelet $\psi_{j, k}(x)$ and the scaling coefficient $\varphi_{j_{0}, k}(x)$, as shown in Equation (1). In this equation, $W_{\phi}\left(j_{0}, k\right)$ and $W_{\psi}\left(j_{0}, k\right)$ are the approximation coefficient and detail coefficient, respectively. The expansion coefficients are shown as follows.

Figure 1 shows a two-step wavelet transformation that generates four sub-bands, where $\psi^{H}$, $\psi^{V}$ and $\psi^{D}$ indicate deviations along the horizontal, vertical, and diagonals edges, respectively. In this diagram, $2 \downarrow$ shows a down stampeding indicator. 2D-DWT can be executed with digital filtration and down samplers. The other sub-bands are generated with discrete 2D scaling functions, with the use of 1D-FWT on $f(x, y)$ [32]. For the computation of the DWT coefficients, we should consider the multiresolution refinement equation, as shown in Equations (6) and (7):

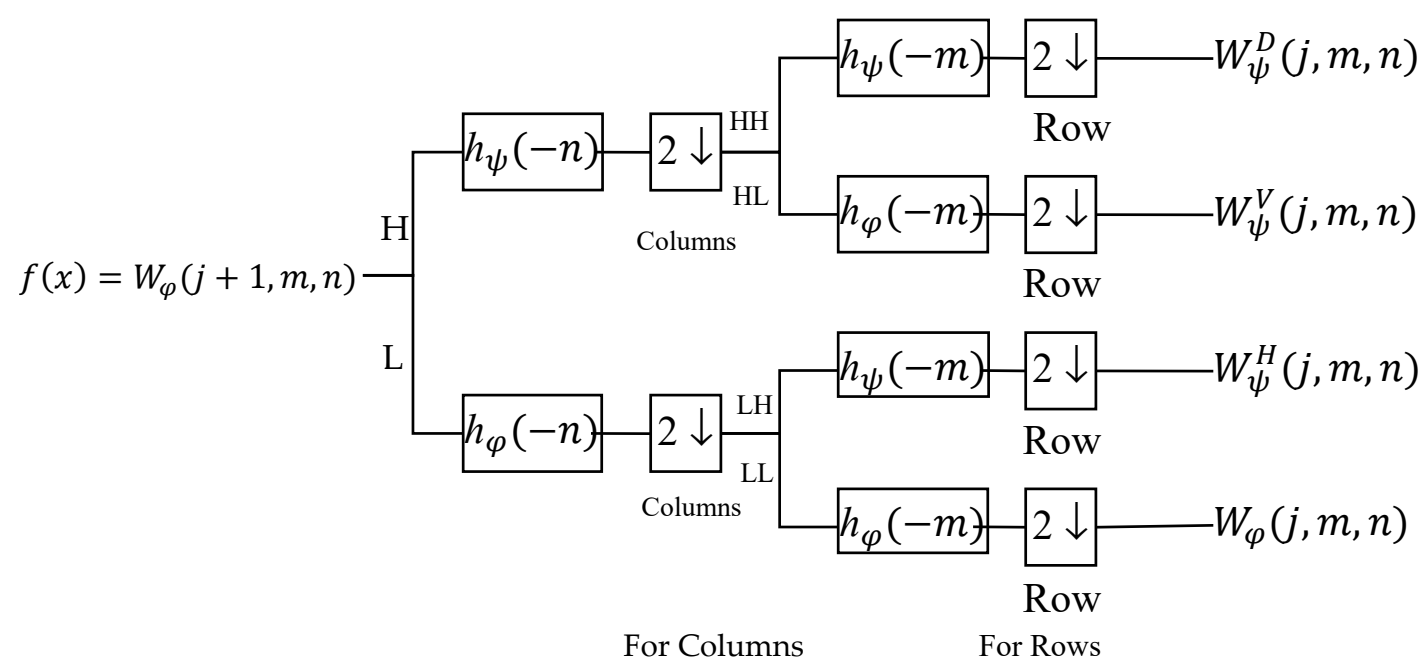

Figure 1. The two-dimensional DWT diagram.

$$
\phi_{j, k}(x)=\sum_{n} 2^{\frac{j}{2}} \phi\left(2^{j} x-n\right) \cdot h_{\phi}(n)
$$




$$
\psi_{j, k}(x)=\sum_{n} 2^{\frac{j}{2}} \psi\left(2^{j} x-n\right) \cdot h_{\psi}(n)
$$

where $h_{\phi}$ and $h_{\psi}$ are the scaling vector and wavelet vector, respectively. $h_{\phi}$ and $h_{\psi}$ may be considered as weights for the summation of Equations (6) and (7). With the inclusion of Equations (6) and (7) into Equations (2) and (3), the following questions result.

$$
\begin{array}{rr}
W_{\phi}(j, k)=h_{\phi}(-n) W_{\phi}(j+1, n), \quad(n=2 k, k \geq 0) \\
W_{\psi}(j, k)=h_{\psi}(-n) W_{\psi}(j+1, n) & j \geq j_{0}
\end{array}
$$

The scaling and wavelet coefficient of a certain scale $j$ may be obtained via the convolution of the scaling coefficients of the next scale $j+1$ (with finer detail) with the order-reversed scaling and wavelet vectors $h_{\phi}(-n)$ and $h_{\psi}(-n)$. Based on Figure 1, the results of the first level of transformation for the column of an input image are as follows:

$$
\begin{aligned}
W_{\phi}(j+1, m, n) & =h_{\phi}(-n) *\left(h_{\phi}(-m) * W_{\phi}^{2 \uparrow}(j, m)+h_{\psi}(-m) * W_{\psi}^{2 \uparrow}(j, m)\right) \\
& +h_{\psi}(-n) *\left(h_{\phi}(-m) * W_{\phi}^{2 \uparrow}(j, m)+h_{\psi}(-m) * W_{\psi}^{2 \uparrow}(j, m)\right), \quad(k \\
& \geq 0) \\
W_{\phi}(j+1, m, n) & =h_{\phi}(-n) h_{\phi}(-m) * W_{\phi}^{2 \uparrow}(j, m)+h_{\phi}(-n) h_{\psi}(-m) * W_{\psi}^{2 \uparrow}(j, m) \\
& +h_{\psi}(-n) h_{\phi}(-m) * W_{\phi}^{2 \uparrow}(j, m)+h_{\psi}(-n) h_{\psi}(-m) * W_{\psi}^{2 \uparrow}(j, m), \quad \quad \quad k \\
& \geq 0) \\
W_{\phi}(j+1, m, n) & =h_{\phi}(-n) h_{\phi}(-m) * W_{\phi}^{2 \uparrow}(j, m)+h_{\phi}(-n) h_{\psi}(-m) * W_{\psi}^{2 \uparrow}(j, m) \\
& +h_{\psi}(-n) h_{\phi}(-m) * W_{\phi}^{2 \uparrow}(j, m)+h_{\psi}(-n) h_{\psi}(-m) * W_{\psi}^{2 \uparrow}(j, m), \quad(k \\
& \geq 0)
\end{aligned}
$$

Generally, 2D- $\varphi(x, y)$, and 3D- $\psi^{H}(x, y), \psi^{V}(x, y)$, and $\psi^{D}(x, y)$ are required to generate a 1D scaling function $\varphi$ and related wavelet $\psi$ [20].

$$
\begin{aligned}
\phi(x, y) & =\phi(x) \phi(y) \\
\psi^{H}(x, y) & =\psi(x) \phi(y) \\
\psi^{V}(x, y) & =\phi(y) \phi(x) \\
\psi^{D}(x, y) & =\psi(x) \psi(y)
\end{aligned}
$$

\subsection{Generalized Autoregressive Conditional Heteroscedasticity}

Bollerslev was the first researcher who developed the GARCH method [33]. It can be considered as being the variance of the time variable, for example, an oscillation. Conditional requires immediate dependence on past observations, and self-control combines past data at the present time. GARCH models are statistical methods that are more common in the economy. Engle [34] presented the process of Autoregressive Conditional Heteroscedasticity (ARCH) to change the conditional variance over time as a factor of past mistakes that remain based on the conditional constant variance. The GARCH process (Algorithm 1) is a general form of $\mathrm{ARCH}$ and is a time series modeling technique that uses the last variance to predict future variances. 


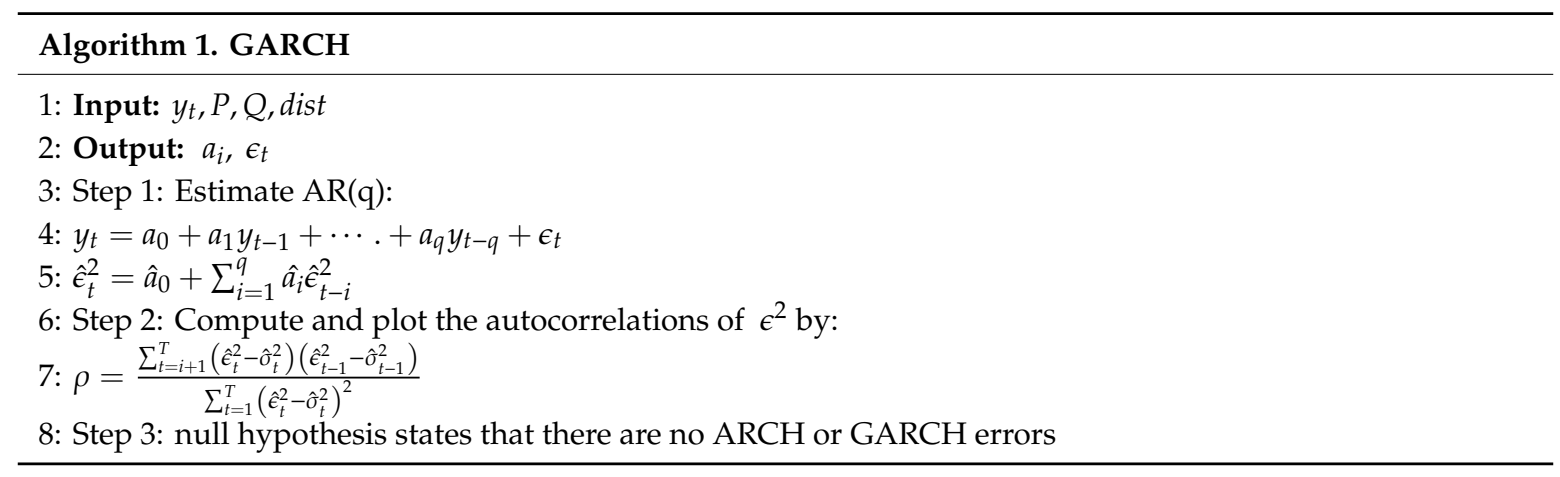

\subsection{Local Linear Approximation}

The Local Linear Approximation is calculated via [35]. In this method, the first and second derivatives are determined so as to generate a fitting function with the observation data.

Let $x$ have three value $x(1), x(2)$, and $x(3)$. An LLA for the derivative of $x$ at the $x(2)$ is calculated via the mean of the two slopes between $x(1)-x(2)$ and between $x(2)-x(3)$, which can now be calculated from $x(3)$ and stored in the matrix $y$ of the same order as $x(3)$ where the $k$ th row of $y$ is:

$$
\begin{gathered}
y_{k 1}=x_{k 2}, \\
y_{k 2}=\frac{x_{k 3}-x_{k 1}}{2 \tau \Delta t}, \\
y_{k 3}=\frac{x_{k 1}-2 x_{k 2}+x_{k 3}}{(\tau \Delta t)^{2}} . \\
\frac{d x(1-\tau)}{d t} \approx \frac{x(1+2 \tau)-x(1)}{2 \tau \Delta t}
\end{gathered}
$$

where the first column of $y$ is the value of $x$ at the moment of measurement indexed in the second column of $x(3)$, and the second and third columns of $y$ are the approximated first and second derivatives, respectively, at that same moment of measurement. In this case, $\tau=1$ since $x(1), x(2)$, and $x(3)$ are successive measures, and $\Delta t$ is the time interval among the measures. The others (for instance $x(1)$, $x(3)$, and $x(5))$ can be calculated with $\tau=2$ being substituted into Equation (20).

\subsection{K-Nearest Neighbour Algorithm}

$\mathrm{KNN}$ is a simple form of machine learning [31,36]. In this algorithm, an article is classified by the values of its neighbors, which are allocated to $\mathrm{k}\left(\in \mathbb{N}^{+}\right)$nearest neighbors [37]. The similarity of each object in a class is utilized as the weight of the class. In the case of a few of the k nearest neighbors sharing a category, the per-neighbor weights of that category are included together at that point, and the obtained weighted entirety is utilized as the probability score of the candidate categories. A positioned list is obtained for the test archive. By thresholding these scores, twofold category assignments are obtained.

\subsection{Proposed Method}

In this paper, we aim to use mathematical methods to diagnose brain diseases. We implemented three methods for the classification and diagnosis of brain tumors (Algorithm 2). The first presented method is Wavelet-GARCH-KNN (WGK). In this method, we first used two-stage 2D-DWT to decompose input images into sub-bands of wavelets. The obtained wavelet coefficients are features of classification. Then, the GARCH model was used for feature extraction with the use of HH1, HL1, LH1, and second-stage HH2, HL2, LH2. Because of the incompatibility of LL with the GARCH model, this sub-band was ignored. To reduce the number of features, the PCA and PCA + LDA method 
was then used, with extracted feature brain lesions being classified with the use of KNN methods. The results are illustrated in the results section.

The second presented method is named Developed Wavelet-GARCH-KNN (D-WGK). In the second method, we overcame the limitation of the WGK algorithm by using homomorphic filtering before a wavelet transformation. Therefore, the LL2 sub-band participated in the GARCH model. Then, similarly to the WGK method, the KNN method was designated for the classification of brain tumors.

The third method is Wavelet-LLA-KNN (WLK). In this method, all sub-bands of wavelet decomposition were used for modeling with the LLA algorithm. The remaining part of the third method was also similar to the WGK and D-WGK method. The results of each algorithm are depicted in the below sections. The structure and proposed model in this study are shown in Figure 2.

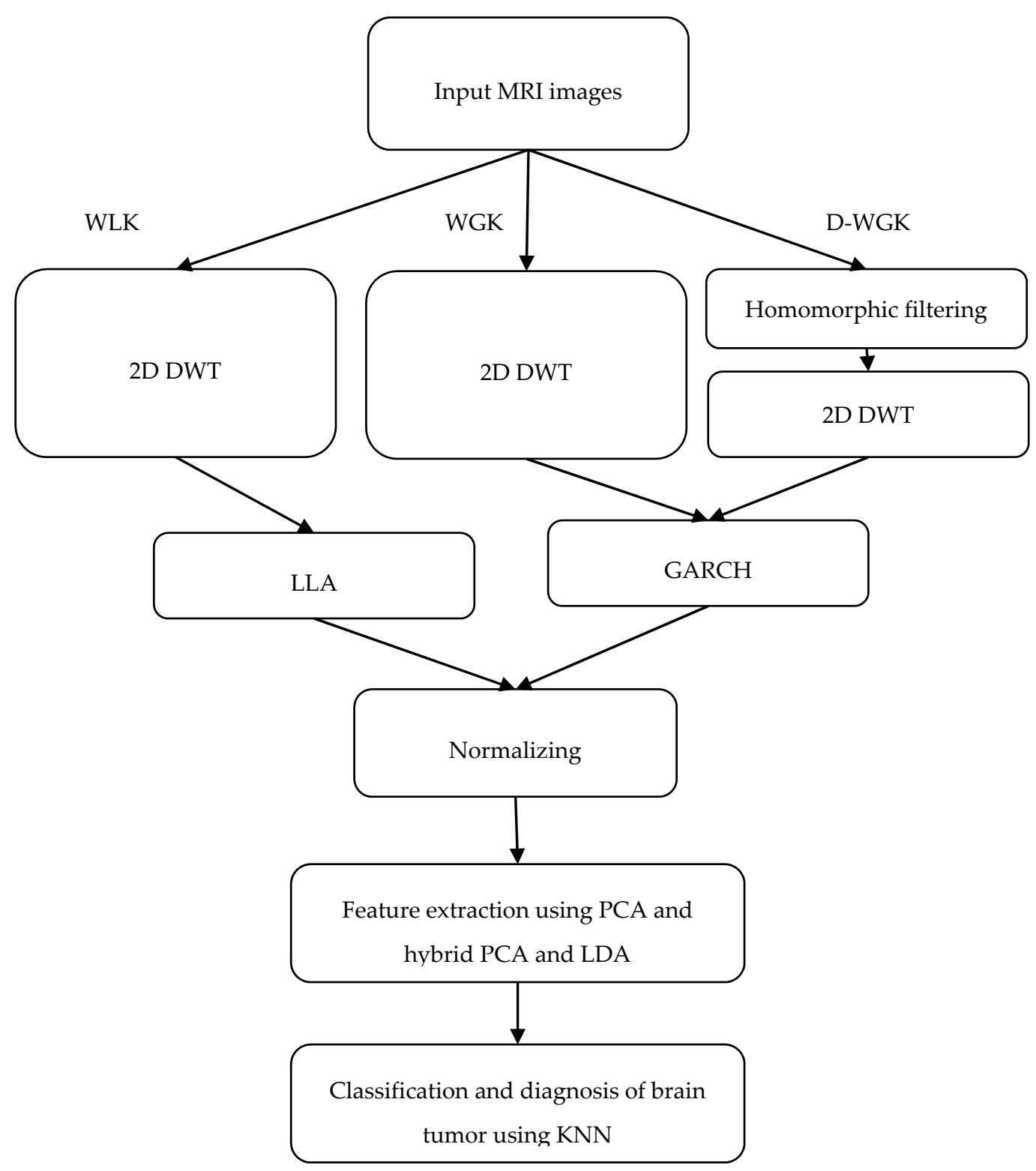

Figure 2. The block diagram of the proposed method. 


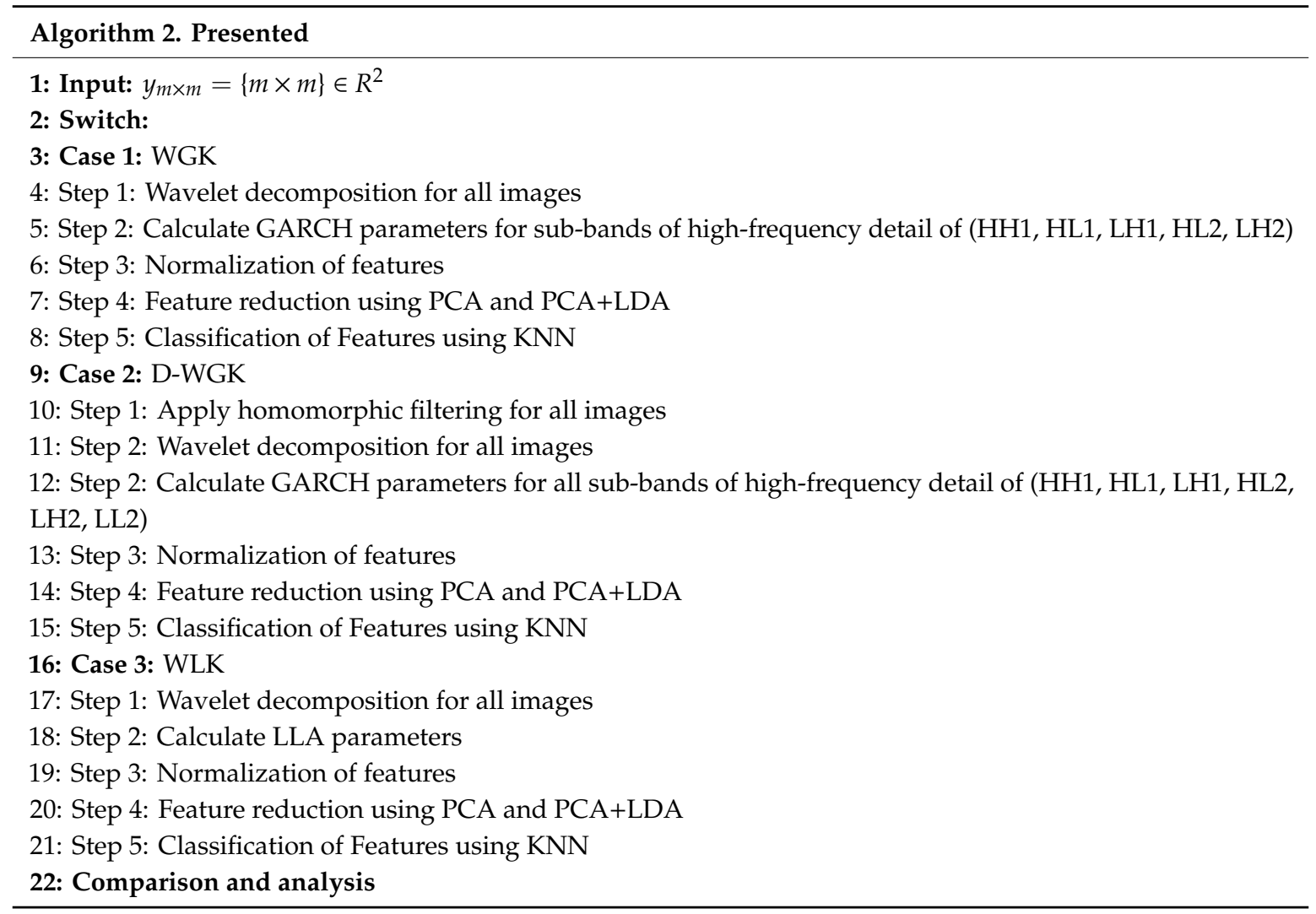

\section{Results and Discussion}

\subsection{Datasets}

In this paper, we used seven brain diseases to implement and test the presented methods. They consist of Alzheimer's, Alzheimer plus visual agnosia, Glioma, Huntington, Meningioma, Pick, and Sarcoma. These diseases, in conjunction with normal brain images, include 240 MRI images from the Harvard medical school website. All images are from T2-weighted MR brain images in the axial plane and have $256 \times 256$ pixels. These images were saved in different folders and studied separately. Therefore, after feature extraction, they were aggregated into a single code folder.

\subsection{Two-dimensional Discrete Wavelet Transforms (2D-DWT)}

In this paper, we used 2D-DWT to separate the sub-bands of images. In this transformation, we input images from $256 \times 256$ pixels to $131 \times 131$ first-stage sub-bands and $69 \times 69$ sub-bands. The example of a wavelet transformation is shown in Figure 3. Additionally, for the second aforementioned method, we needed homomorphic filtering. 


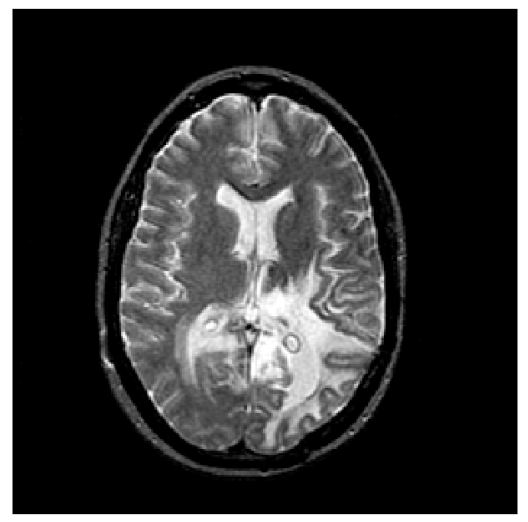

First Transformation

$\mathrm{L}$

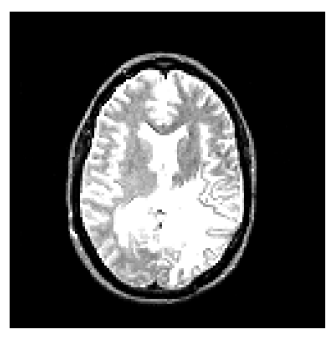

$\mathrm{H}$

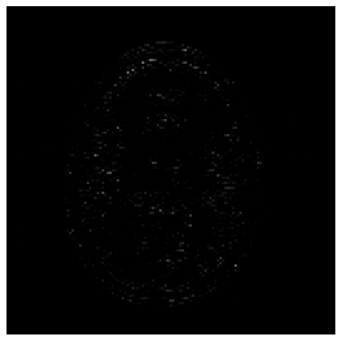

Second Transformation

$\mathrm{H}$
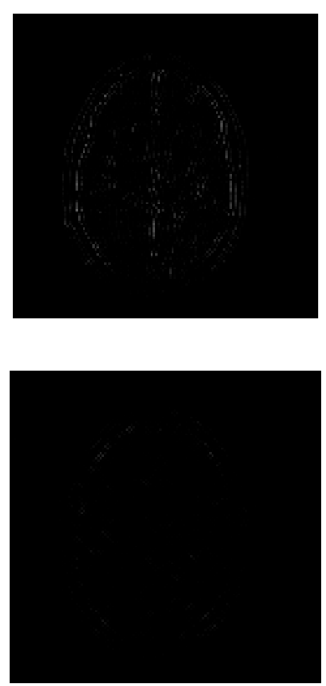

$\mathrm{L}$
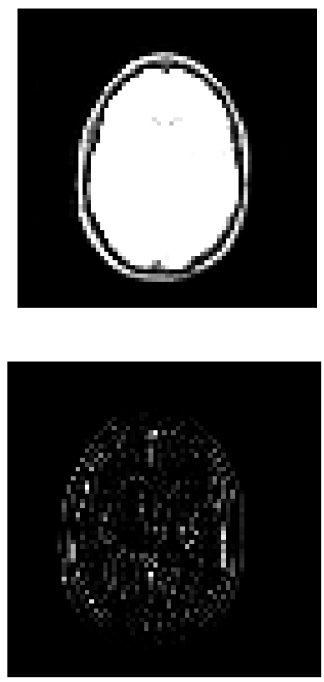

$\mathrm{H}$
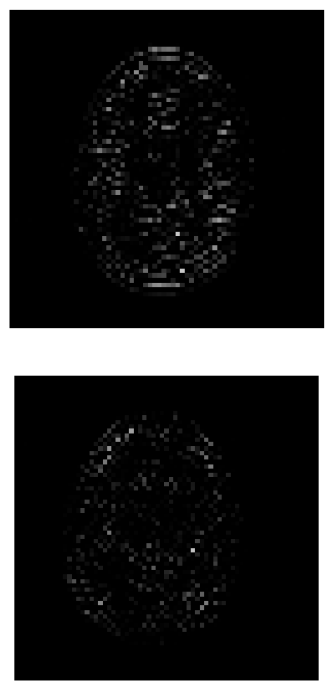

Figure 3. The sub-bands of the wavelet transformation.

The results of the wavelet discretization when using homomorphic filtering $(\sigma=5)$ are shown in Figure 4. The top images show the original image without (with) the filtration, and the first and second transformation are shown on the left and right sides of the images, respectively. Regarding the literature studies, the GARCH model was not compatible with LL2 sub-bands [38]. This situation is obviously shown in Figure 3 (LL2). Because all the brain sections of the images were almost within the GARCH model in $(1,1)$, we did not find the model coefficient to be significant for the GARCH $(1,1)$ model. In this paper, we overcame this limitation and made the LL2 model be compatible with the GARCH $(1,1)$ model. To overcome this condition, we used homomorphic filtration for the main image, and then the 2D-DWT was performed on it. With this method, we increased the contrast of the LL2 sub-band, as can be seen in Figure 4 (LL2). 


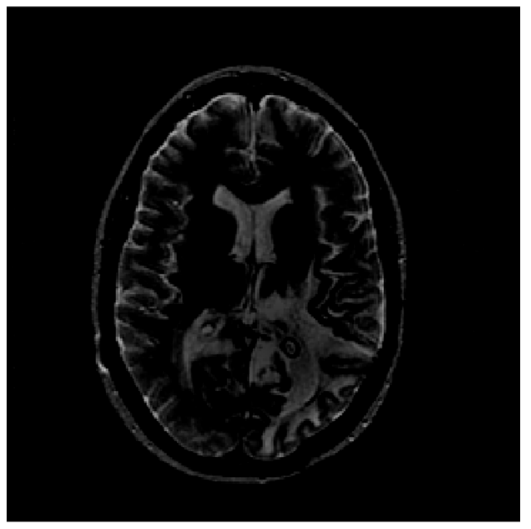

First Transformation

$\mathrm{L}$
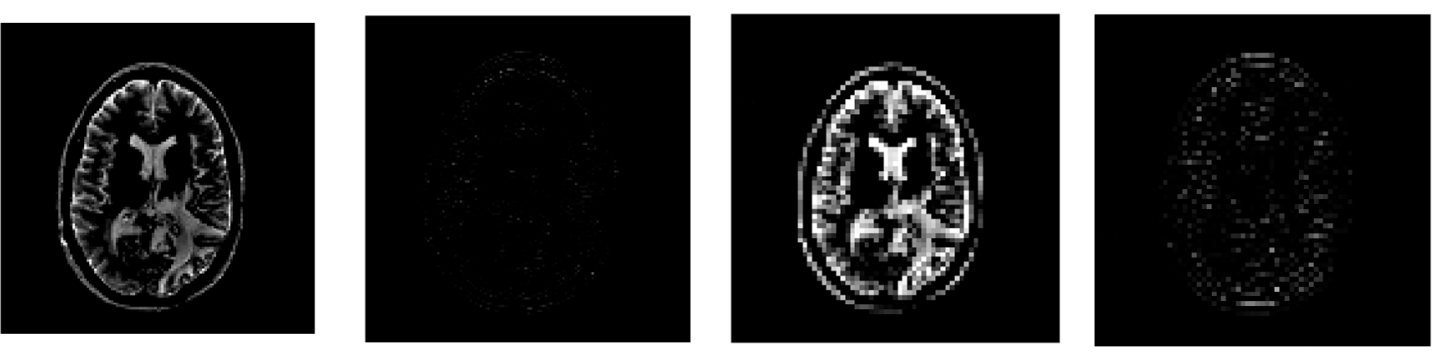

$\mathrm{H}$
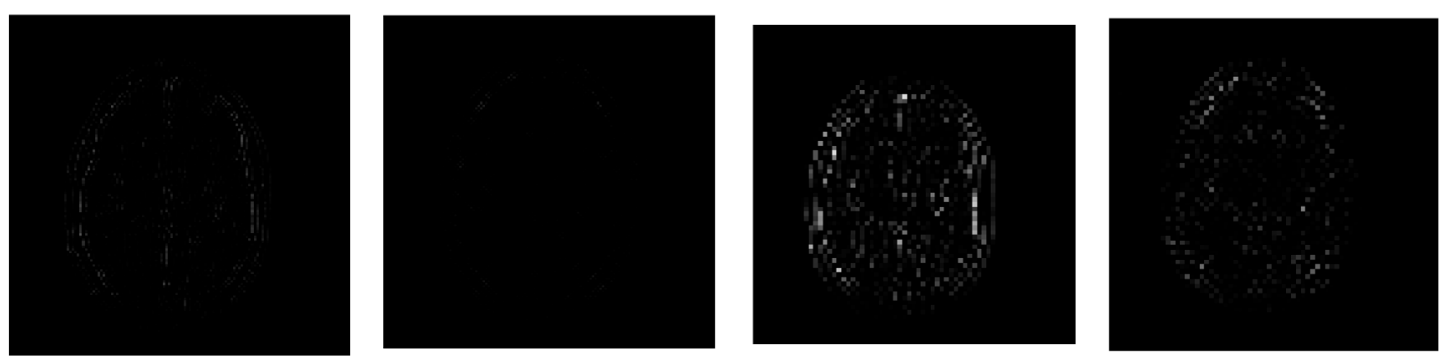

Figure 4. The sub-bands of the wavelet transformation using homomorphic filtering.

\subsection{Feature Reduction}

In this section, we used nonconvulsive status epilepticus (NCSE) to extract features and classify them. Via this method, we can classify the features into two classification states: two-classes and eight-classes. In the two-class state, we classify the features into two classes to diagnosis the normal and abnormal MRI images. Using this state, we can find patient and inpatient brain images. Moreover, using the eight-class state, we can classify the brain images into seven different classes in conjunction with normal brain images.

In this paper, we studied different methods to reduce features, consisting of:

WGK: Using GARCH without LL2 + PCA

WGK: Using GARCH without LL2 + PCA + LDA

D-WGK: Using Homomorphic filtering + GARCH with LL2 + PCA 
WLK: Using LLA + PCA

WLK: Using LLA + PCA + LDA

The results are depicted in Figures 5-8. Figure 5 shows the feature reduction plots used to find the best number of classes. In this figure, we used two methods, 2D-DWT and GARCH $(1,1)$, used with and without LL2 sub-bands. The result shows that with the addition of LL2 to the GARCH method, the model is developed. Furthermore, the results of the PCA method show that we can use 14 features for the classification of images.

Furthermore, this enhancement is shown in Figure 6 for the two-class state. Additionally, in this state, the method is developed, and a number of features are decremented from 6 to 5 . This can speed up the classification method and increase the accuracy of the methods because we used all sub-bands of the 2D-DWT method for classification with fewer features.

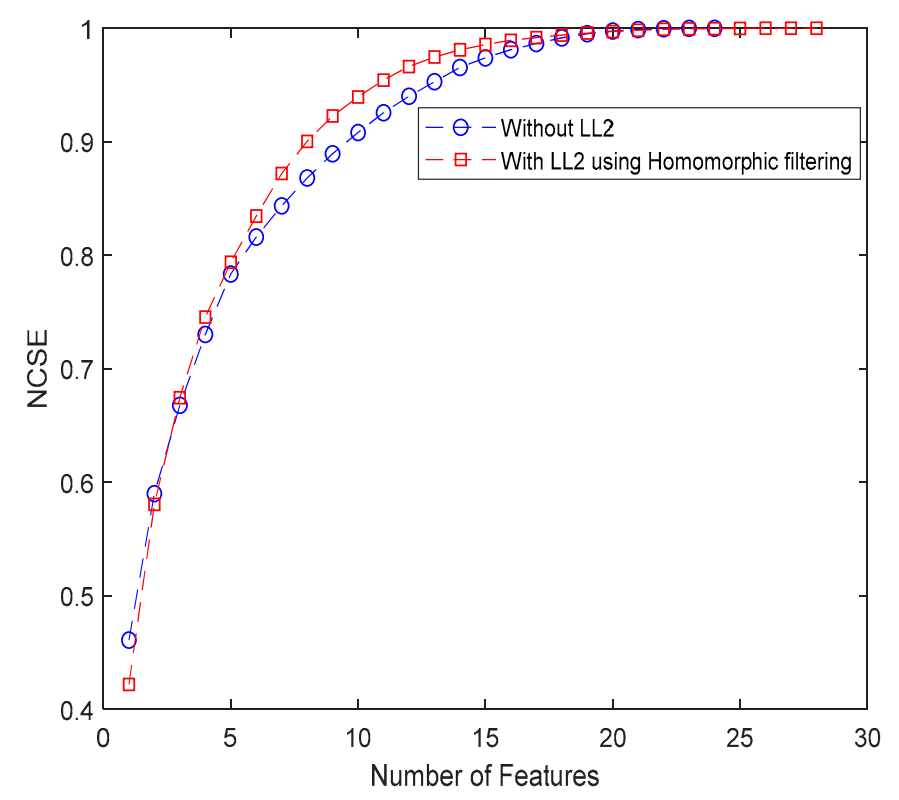

Figure 5. Normalized cumulative summation of eigenvalues of training data for GARCH $(1,1)$ with and without LL2 (Eight-classes).

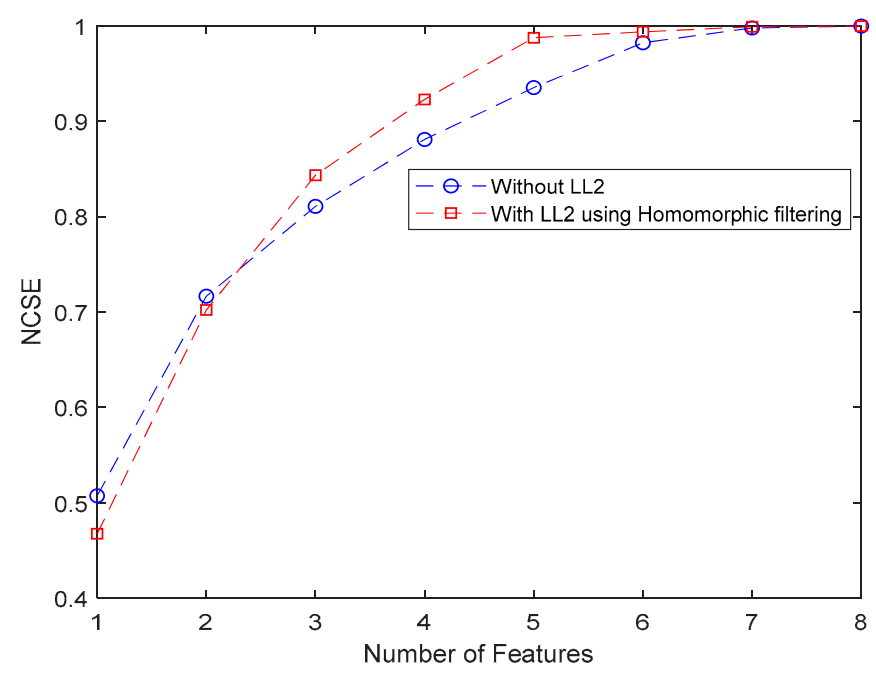

Figure 6. Normalized cumulative summation of eigenvalues of training data for GARCH $(1,1)$ with and without LL2 (two-classes). 


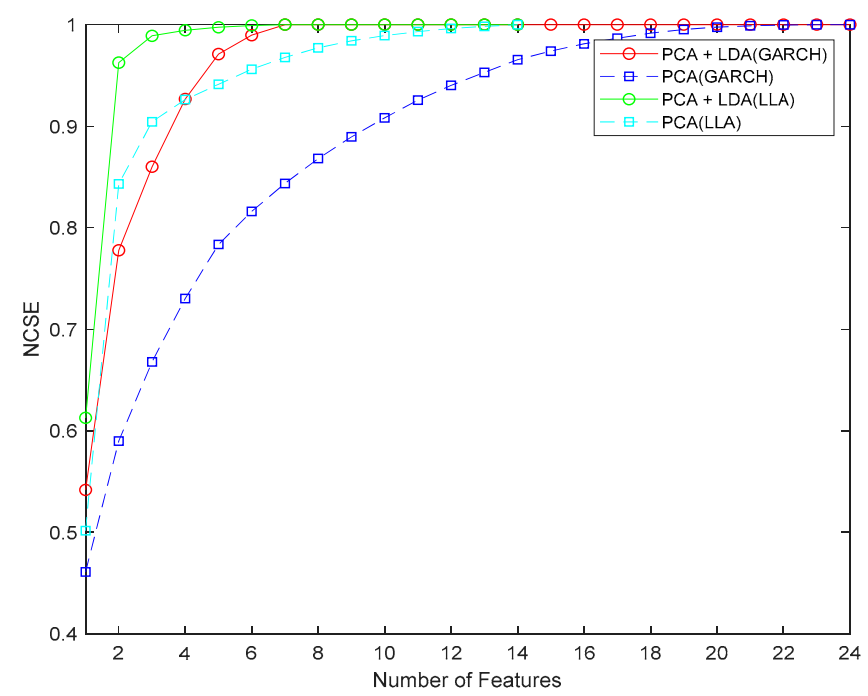

Figure 7. Normalized cumulative summation of eigenvalues of training data for the GARCH and LLA methods for different PCA and PCA + LDA methods (eight-classes).

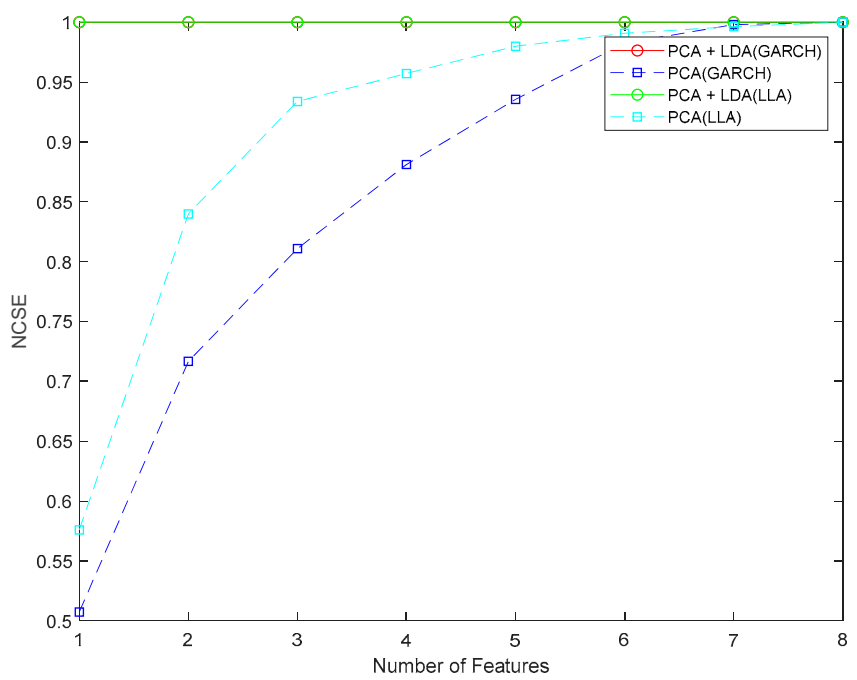

Figure 8. Normalized cumulative summation of eigenvalues of training data for the GARCH and LLA methods for different PCA and PCA + LDA methods (two-classes).

Figures 7 and 8 show the feature reduction results for the presented LLA and GARCH model using the PCA and PCA + LDA methods. In the eight-class method (Figure 7), the best number of features for the GARCH and PCA method was 20, which for GARCH + PCA + LLDA decreased to 10 features. Using the presented method, the LLA + PCA method's number of features decreased to 7 features. Furthermore, for the LLA + PCA + LDA method, the best number of features should be three features. The results showed that the last presented method decreased the number of features to 3 so that it would be great for feature reduction.

For a two-class state (Figure 8), this reduction is conspicuously shown. The resolution between the classes is high, which indicates the ability of LLA + PCA + LDA in incremental inter-class distances and decremental intra-class distances. 


\subsection{The Classification Results}

In this paper, we used the k-Nearest Neighborhood (KNN) method to classify the input features. $\mathrm{KNN}$ is a non-parametric method used in data mining, machine learning, and pattern recognition. The KNN algorithm is one of the ten most used algorithms in various machine learning and data mining projects in the industry. The KNN algorithm can be used for classification and regression issues. However, it is often used for classification issues.

The value of $\mathrm{K}$ in the KNN method is one of the effective parameters in classification. The mean classification accuracy was determined for different values of $K$, which was increased from 1 to 11 in steps of two for both states. The results are depicted in Figure 9. The results show that, for $\mathrm{K} \leq 5$, the classifier has good efficiency. Furthermore, the accuracy of the LLA method in the KNN classifier is greater than the GARCH method.

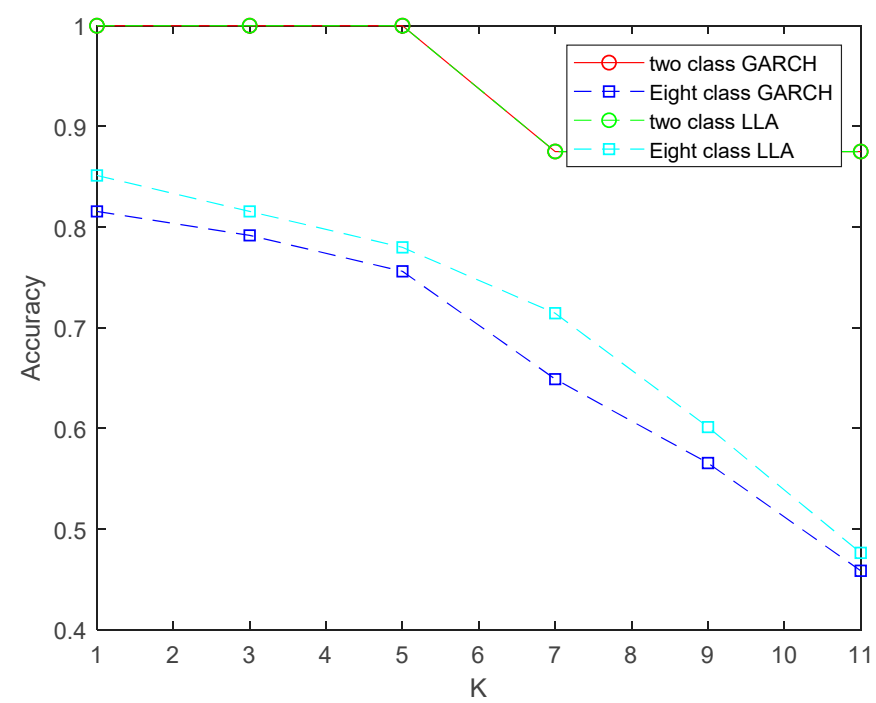

Figure 9. The plots of the accuracy of the two- and eight-class methods for different $\mathrm{K}$ values.

In the statistics, indicators of sensitivity and specificity are utilized to evaluate the result of the binary classification (two-class). When the data can be divided into positive and negative groups, the accuracy of the results of a test that divides the information into these two categories is measurable and describable using sensitivity and attribute indicators. Sensitivity means a proportion of positive cases that will test them correctly as being positive. Specificity means the proportion of negative cases that mark them correctly as being negative.

True Positive (TP): The disease is diagnosed correctly.

False Positive (FP): A healthy person is diagnosed with mistakes.

True Negative (TN): A healthy person is diagnosed correctly.

False Negative (FN): The disease is diagnosed with mistakes.

The sensitivity parameter is calculated by dividing the numbers of TP cases by the sum of TP cases and FN cases.

$$
\text { sensitivity, } \mathrm{TPR}=\frac{\mathrm{TP}}{\mathrm{TP}+\mathrm{FN}}
$$

In a similar way, the specificity results are the division of TN cases by the sum of FP and TN cases.

$$
\text { specificity, } \mathrm{TNR}=\frac{\mathrm{TN}}{\mathrm{TN}+\mathrm{FP}}
$$

Other classification criteria, such as precision, accuracy, and fall-out, are defined as following Equations (23)-(25).

$$
\text { precision, } \mathrm{PPV}=\frac{\mathrm{TP}}{\mathrm{TP}+\mathrm{FP}}
$$




$$
\begin{gathered}
\operatorname{accuracy}(\mathrm{ACC})=\frac{\mathrm{TP}+\mathrm{TN}}{\mathrm{TP}+\mathrm{TN}+\mathrm{FP}+\mathrm{FN}} \\
\text { fall }-\operatorname{out}(\mathrm{FPR})=\frac{\mathrm{FP}}{\mathrm{FP}+\mathrm{FN}}
\end{gathered}
$$

The results of the classification using the LLA method are shown in Tables 1 and 2. Table 1 shows the results of the classification using the presented methods. The results show that the maximum accuracy belongs to the presented LLA method that conducted the extraction using the combination of the PCA and LDA methods. Moreover, the minimum one belongs to the GARCH method that used the PCA feature extraction method.

Table 1. The comparison between the presented methods.

\begin{tabular}{ccccc}
\hline Method & Class & Images & Features & Accuracy \\
\hline Ref. $^{*}$ & 6 & 56 & 6 & 91.5 \\
PCA + LDA (WGK) ** & 8 & 80 & 10 & 89.4 \\
PCA (WGK) ** & 8 & 80 & 22 & 90.1 \\
Proposed PCA + LDA (D-WGK) & 8 & 240 & 10 & 90.2 \\
Proposed PCA (D-WGK) & 8 & 240 & 20 & 89.3 \\
Proposed PCA + LDA (WLK) & 8 & 240 & 3 & 92.5 \\
Proposed PCA (WLK) & 8 & 240 & 7 & 91.3 \\
\hline
\end{tabular}

* Marti nez, et al. [39]; ** Kalbkhani, Shayesteh and Zali-Vargahan [38].

Table 2. The results of the classification for eight-class states using PCA + LDA (WLK).

\begin{tabular}{cccccc}
\hline Diseases & TPR & TNR & PPV & ACC & FPR \\
\hline Alzheimer & 0.933 & 1 & 0.903 & 0.967 & 0 \\
Alzheimer+ & 0.933 & 1 & 0.875 & 0.967 & 0 \\
Glioma & 0.900 & 1 & 1 & 0.950 & 0 \\
Huntington & 0.967 & 1 & 0.906 & 0.983 & 0 \\
Meningioma & 0.967 & 1 & 1 & 0.983 & 0 \\
Pick & 0.867 & 1 & 0.839 & 0.933 & 0 \\
Sarcoma & 0.833 & 1 & 0.926 & 0.917 & 0 \\
\hline
\end{tabular}

Therefore, we can prioritize the presented methods that follow a maximum sensitivity and minimum fall-out as PCA + LDA (WLK), PCA (WLK), PCA + LDA (WGK), and PCA (WGK) (see Figure 10). This shows that the LLA method is better than the GARCH model in terms of robustness, sensitivity, and accuracy. Moreover, the combination of PCA and LDA produces better results than single PCA. One of the main reasons that the GARCH model has not produced a good model is the incompatibility of this method with some images. Table 2 also shows the results of a classification in the eight-class state, and the results show the acceptable outperformance of most diseases. The diagnosis of Pick and Sarcoma is somewhat more inaccurate than that of others; this is because of the complex images of these diseases.

Figure 11 shows the confusion matrix of the presented model of the hybrid PCA, LDA, and LLA methods for the diagnosis of normal and abnormal images. The main diameter of the matrix shows the number of images detected correctly. From 210 abnormal images, 18 (8.57\%) were recognized as normal lesions. However, 192 (91.43\%) of the abnormal images were diagnosed correctly. Nevertheless, all the normal images were detected, and $92.5 \%$ (accuracy) of all images are were correctly classified, while $7.5 \%$ were incorrectly classed. 


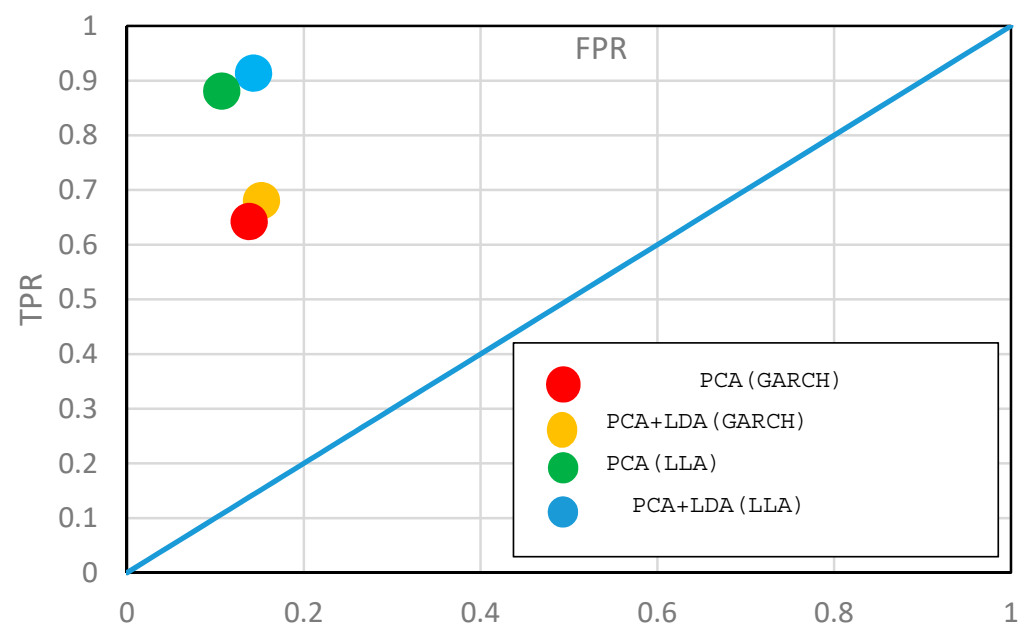

Figure 10. The Receiver Operating Characteristic (ROC) curve of the presented method.

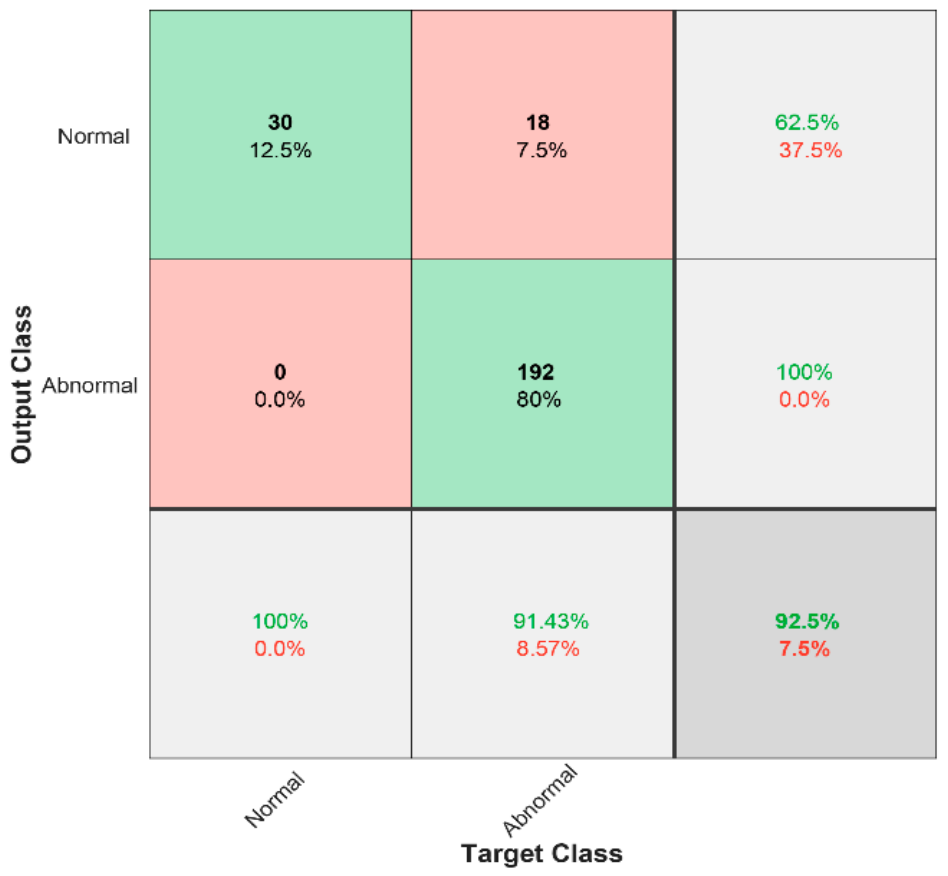

Figure 11. The confusion matrix for two-classes states using PCA-LDA (LLA).

Figure 12 also shows the confusion matrix of the presented method for the classification in eight classes. The lower row of the matrix shows the percentages of each disease that were detected correctly (sensitivity). The maximum detection percentage belonged to normal images, and then Huntington and Meningioma came second. However, only $93.3 \%$ of Sarcomas were diagnosed correctly. In the end, $92.5 \%$ (accuracy) of all images were classified in the proper class, while $7.3 \%$ of them could not be recognized and were incorrectly classed. The red cells show incorrect choices or false ones. In each column, the sum of the elements equals the number of images of each disease. For example, for Alzheimer's (first column), 28 images (from 30) were diagnosed correctly; however, two images were classified into the Alzheimer plus category. 


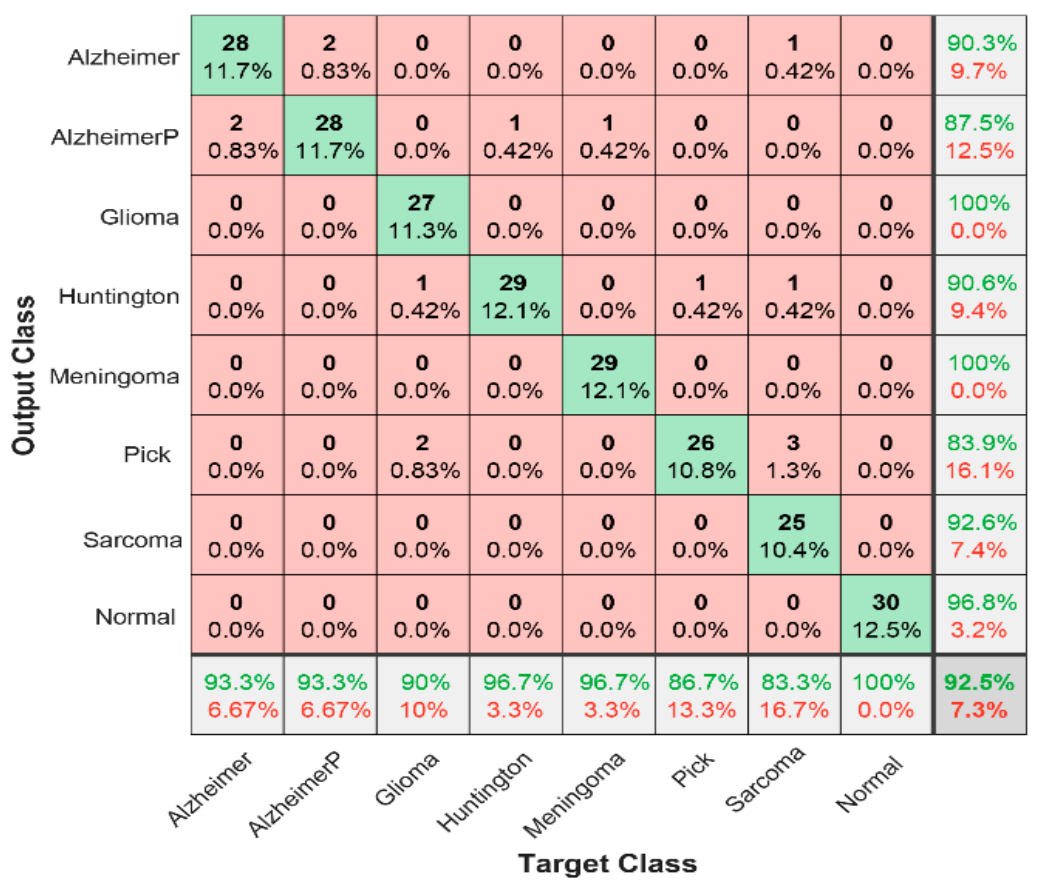

Figure 12. The confusion matrix for eight-classes states using PCA-LDA (WLK).

\section{The Complexity Analysis}

In the proposed method, we used five major approaches. Therefore, we should calculate their complexity. The complexity of PCA is $O\left(\min \left(p^{3}, n^{3}\right)\right)$, where $p$ shows the number of features, and $n$ is the data abundance (image size $256 \times 256$ ) [40]. Additionally, the complexity of the LDA method for feature extraction is $O\left(n p^{2}\right)$ if $n>p$. Otherwise, it is $O\left(p^{3}\right)$. The complexity of 2D-DWT is $O\left(4 M n^{2} \log 2 n\right)$, where $M$ is the number of vanishing moments of the mother wavelets that are used. The complexity of the GARCH $(1,1)$ method depends on the autocorrelation complexity and is $O(n)$, where, in this case, $n$ is $256 \times 256$. Regarding the complexity of the LLA method, we can calculate this as $O\left(n^{\prime} n\right)$, where $n^{\prime}$ is of the order of the derivative in the method and where, in this case, $n^{\prime}=2$. The complexity of the KNN method is $O(n p k)$, where, in this case, $k=1$. Therefore, the complexity of the presented method is as follows: PCA $(\mathrm{GARCH})$ is $O\left(\min \left(p^{3}, n^{3}\right)+n\right), \mathrm{PCA}+\mathrm{LDA}(\mathrm{GARCH})$ is $O\left(\min \left(p^{3}, n^{3}\right)+n p^{2}+n\right)$, PCA (LLA) is $O\left(\min \left(p^{3}, n^{3}\right)+2 n\right)$, and PCA + LDA (LLA) is $O\left(\min \left(p^{3}, n^{3}\right)+n p^{2}+2 n\right)$; therefore, the group of the LLA method is somewhat more complex than that of the GARCH group; however, the result is remarkable and compatible with all of the images.

\section{Conclusions}

In this paper, a hybrid algorithm for determining the diagnosis of brain disease in MRIs is presented. Initially, the two-level transformation of the 2D-DWT was calculated as the input images. The sub-banded wavelet coefficients could be modeled using the GARCH and LLA models. We used five studies in this paper. After using the 2D-DWT method and the separation of the image into six sub-bands to model the sub-bands, we used GARCH $(1,1)$ without using the Low-Low sub-band in the second wavelet level (use of WGK). Because this sub-band was incompatible with the GARCH $(1,1)$ method in terms of overcoming this condition, we used homomorphic filtering before 2D-DWT (use of D-WGK). The results showed that, by using Homomorphic filtering, the LL2 sub-band with the maximum image data could be utilized in the $\operatorname{GARCH}(1,1)$ method with high performance. Moreover, we used the LLA method to model the 2D-DWT sub-bands. In this method, we used all of the sub-bands to model features (use of WLK). The results showed that using the LLA method, we could reduce the number of features from 20 to 3 . Then, we classified the images using the KNN method. The results demonstrated the high accuracy and robustness of the presented methods. The results 
showed that the WLK method was better than the WGK and D-WGL models in terms of robustness, sensitivity, and accuracy. Furthermore, the hybrid of PCA and LDA produced better results than PCA. One of the main reasons why the GARCH model has not produced a good model relates to the incompatibility of this method with some images. We overcame this problem in D-WGT with the use of homomorphic filtering. The results of an eight-class classification (diagnosis of disease type) showed an acceptable outperformance for most diseases. The diagnosis of Pick and Sarcoma was somewhat more inaccurate than that of the others; this is because of the complex images of these diseases. Out of the abnormal images, $8.57 \%$ were recognized as normal lesions. However, $91.43 \%$ of abnormal images were diagnosed correctly. Nevertheless, all the normal images were detected with 92.5\% accuracy. The maximum detection percentage belonged to normal images, and then Huntington and Meningioma came second. However, $93.3 \%$ of sarcomas were classified correctly. In the end, $92.5 \%$ of all images were classified in their proper class, with a 7.3\% error. Future work should focus on increasing the dataset volume for the diagnosis of brain tumors. Furthermore, it could be implemented for other MRI images, like breast cancer, prostate cancer, and so on. The novel methods of deep learning could also be enriched with this feature extraction method, which increases process speed and accuracy.

Author Contributions: Conceptualization, A.H.; Formal analysis, A.H. and S.J.G.; Methodology, A.H. and V.B.; Project administration, S.J.G. and V.B.; Resources, A.H. and A.M.; Software, A.H.; Supervision, S.J.G.; Validation, A.H.; Visualization, S.J.G. and V.B.; Writing—original draft, A.M.; Writing—review \& editing, A.M. All authors have read and agreed to the published version of the manuscript.

Funding: The funding sources had no involvement in the study design, collection, analysis or interpretation of data, writing of the manuscript or in the decision to submit the manuscript for publication.

Conflicts of Interest: The authors declare no conflict of interest.

Ethical Approval: This article does not contain any studies with human participants or animals performed by any of the authors.

\section{References}

1. Herszterg, I.; Poggi, M.; Vidal, T. Two-Dimensional Phase Unwrapping via Balanced Spanning Forests. INFORMS J. Comput. 2019, 31, 527-543. [CrossRef]

2. Won, D.; Manzour, H.; Chaovalitwongse, W. Convex Optimization for Group Feature Selection in Networked Data. INFORMS J. Comput. 2020, 32, 182-198. [CrossRef]

3. Zhang, Y.; Dong, Z.; Wu, L.; Wang, S. A hybrid method for MRI brain image classification. Expert Syst. Appl. 2011, 38, 10049-10053. [CrossRef]

4. Abdullah, N.; Ngah, U.K.; Aziz, S.A. Image classification of brain MRI using support vector machine. In Proceedings of the 2011 IEEE International Conference on Imaging Systems and Techniques, Penang, Malaysia, 17-18 May 2011; pp. 242-247.

5. Gillis, N.; Vavasis, S.A. On the Complexity of Robust PCA and $\ell 1$-Norm Low-Rank Matrix Approximation. Math. Oper. Res. 2018, 43, 1072-1084. [CrossRef]

6. Abdulkareem, M.; Bakhary, N.; Vafaei, M.; Noor, N.M.; Mohamed, R.N. Application of two-dimensional wavelet transform to detect damage in steel plate structures. Measurement 2019, 146, 912-923. [CrossRef]

7. Talo, M.; Baloglu, U.B.; Yıldırım, Ö.; Rajendra Acharya, U. Application of deep transfer learning for automated brain abnormality classification using MR images. Cogn. Syst. Res. 2019, 54, 176-188. [CrossRef]

8. Abdelaziz Ismael, S.A.; Mohammed, A.; Hefny, H. An enhanced deep learning approach for brain cancer MRI images classification using residual networks. Artif. Intell. Med. 2020, 102, 101779. [CrossRef]

9. Chaplot, S.; Patnaik, L.M.; Jagannathan, N.R. Classification of magnetic resonance brain images using wavelets as input to support vector machine and neural network. Biomed. Signal. Process. Control 2006, 1, 86-92. [CrossRef]

10. Hackmack, K.; Paul, F.; Weygandt, M.; Allefeld, C.; Haynes, J.D. Multi-scale classification of disease using structural MRI and wavelet transform. Neuroimage 2012, 62, 48-58. [CrossRef]

11. Maitra, M.; Chatterjee, A. Hybrid multiresolution Slantlet transform and fuzzy c-means clustering approach for normal-pathological brain MR image segregation. Med. Eng. Phys. 2008, 30, 615-623. [CrossRef] 
12. Ramathilagam, S.; Pandiyarajan, R.; Sathya, A.; Devi, R.; Kannan, S.R. Modified fuzzy c-means algorithm for segmentation of T1-T2-weighted brain MRI. J. Comput. Appl. Math. 2011, 235, 1578-1586. [CrossRef]

13. Rivest-Hénault, D.; Cheriet, M. Unsupervised MRI segmentation of brain tissues using a local linear model and level set. Magn. Reson. Imaging 2011, 29, 243-259. [CrossRef] [PubMed]

14. Hussain, S.J.; Savithri, A.S.; Devi, P.V.S. Segmentation of brain MRI with statistical and 2D wavelet features by using neural networks. In Proceedings of the 3rd International Conference on Trendz in Information Sciences \& Computing (TISC2011), Chennai, India, 8-9 December 2011; pp. 154-159.

15. Bhattacharyya, D.; Kim, T.-H. Brain Tumor Detection Using MRI Image Analysis; Springer: Berlin/Heidelberg, Germany, 2011; pp. 307-314.

16. Kim, H.T.; Kim, B.Y.; Park, E.H.; Kim, J.W.; Hwang, E.W.; Han, S.K.; Cho, S. Computerized recognition of Alzheimer disease-EEG using genetic algorithms and neural network. Future Gener. Comput. Syst. 2005, 21, 1124-1130. [CrossRef]

17. Abásolo, D.; Hornero, R.; Espino, P.; Poza, J.; Sánchez, C.I.; de la Rosa, R. Analysis of regularity in the EEG background activity of Alzheimer's disease patients with Approximate Entropy. Clin. Neurophysiol. 2005, 116, 1826-1834. [CrossRef] [PubMed]

18. Gholipour, A.; Estroff, J.A.; Barnewolt, C.E.; Connolly, S.A.; Warfield, S.K. Fetal brain volumetry through MRI volumetric reconstruction and segmentation. Int. J. Comput. Assist. Radiol. Surg. 2011, 6, 329-339. [CrossRef]

19. Zacharaki, E.I.; Kanas, V.G.; Davatzikos, C. Investigating machine learning techniques for MRI-based classification of brain neoplasms. Int. J. Comput. Assist. Radiol. Surg. 2011, 6, 821-828. [CrossRef] [PubMed]

20. Fritzsche, K.H.; Stieltjes, B.; Schlindwein, S.; van Bruggen, T.; Essig, M.; Meinzer, H.P. Automated MR morphometry to predict Alzheimer's disease in mild cognitive impairment. Int. J. Comput. Assist. Radiol. Surg. 2010, 5, 623-632. [CrossRef] [PubMed]

21. Devanand, D.P.; Bansal, R.; Liu, J.; Hao, X.; Pradhaban, G.; Peterson, B.S. MRI hippocampal and entorhinal cortex mapping in predicting conversion to Alzheimer's disease. Neuroimage 2012, 60, 1622-1629. [CrossRef] [PubMed]

22. Zöllner, F.G.; Emblem, K.E.; Schad, L.R. SVM-based glioma grading: Optimization by feature reduction analysis. Z. Med. Phys. 2012, 22, 205-214. [CrossRef] [PubMed]

23. Afshar, P.; Mohammadi, A.; Plataniotis, K.N. Brain Tumor Type Classification via Capsule Networks. In Proceedings of the 2018 25th IEEE International Conference on Image Processing (ICIP), Athens, Greece, 7-10 October 2018; pp. 3129-3133.

24. Mohan, G.; Subashini, M.M. MRI based medical image analysis: Survey on brain tumor grade classification. Biomed. Signal Process. Control 2018, 39, 139-161. [CrossRef]

25. Huang, H.; Meng, F.; Zhou, S.; Jiang, F.; Manogaran, G. Brain Image Segmentation Based on FCM Clustering Algorithm and Rough Set. IEEE Access 2019, 7, 12386-12396. [CrossRef]

26. Breton, M.; Frutos, J.d. Option Pricing Under GARCH Processes Using PDE Methods. Oper. Res. 2010, 58, 1148-1157. [CrossRef]

27. Zhao, Y.-B.; Luo, Z.-Q. Constructing New Weighted $\ell 1$-Algorithms for the Sparsest Points of Polyhedral Sets. Math. Oper. Res. 2016, 42, 57-76. [CrossRef]

28. Milstein, A.; Topol, E.J. Computer vision's potential to improve health care. Lancet 2020, 395, 1537. [CrossRef]

29. Liu, D.; Oczak, M.; Maschat, K.; Baumgartner, J.; Pletzer, B.; He, D.; Norton, T. A computer vision-based method for spatial-temporal action recognition of tail-biting behaviour in group-housed pigs. Biosyst. Eng. 2020, 195, 27-41. [CrossRef]

30. Burrus, C.S.; Gopinath, R.A. Introduction to Wavelets and Wavelet Transforms: A Primer; Perason Prentice Hall: Upper Saddle River, NJ, USA, 1998.

31. Bollerslev, T. Generalized autoregressive conditional heteroskedasticity. J. Econom. 1986, 31, 307-327. [CrossRef]

32. Engle, R.F. Autoregressive Conditional Heteroscedasticity with Estimates of the Variance of United Kingdom Inflation. Econometrica 1982, 50, 987-1007. [CrossRef]

33. Boker, S.M. Differential Models and "Differential Structural Equation Modeling of Intraindividual Variability"; American Psychological Association: 2001. Available online: https://psycnet.apa.org/record/2001-01077-006 (accessed on 2 June 2020).

34. Lutu, P.E.N.; Engelbrecht, A.P. Base Model Combination Algorithm for Resolving Tied Predictions for K-Nearest Neighbor OVA Ensemble Models. INFORMS J. Comput. 2012, 25, 517-526. [CrossRef] 
35. Fukunaga, K.; Narendra, P.M. A Branch and Bound Algorithm for Computing k-Nearest Neighbors. IEEE Trans. Comput. 1975, C-24, 750-753. [CrossRef]

36. Kalbkhani, H.; Shayesteh, M.G.; Zali-Vargahan, B. Robust algorithm for brain magnetic resonance image (MRI) classification based on GARCH variances series. Biomed. Signal. Process. Control 2013, 8, 909-919. [CrossRef]

37. Marti-nez, J.M.P.; Berlanga, R.; Aramburu, M.J.; Pedersen, T.B. Integrating Data Warehouses with Web Data: A Survey. IEEE Trans. Knowl. Data Eng. 2008, 20, 940-955. [CrossRef]

38. Johnstone, I.M.; Lu, A.Y. On Consistency and Sparsity for Principal Components Analysis in High Dimensions. J. Am. Stat. Assoc. 2009, 104, 682-693. [CrossRef] [PubMed]

39. Shi, Q.; Zhang, H. Fault diagnosis of an autonomous vehicle with an improved SVM algorithm subject to unbalanced datasets. IEEE Trans. Ind. Electron. 2020. [CrossRef]

40. Qi, Z.; Shi, Q.; Zhang, H. Tuning of digital PID controllers using particle swarm optimization algorithm for a CAN-based DC motor subject to stochastic delays. IEEE Trans. Ind. Electron. 2019, 67, 5637-5646. [CrossRef]

(C) 2020 by the authors. Licensee MDPI, Basel, Switzerland. This article is an open access article distributed under the terms and conditions of the Creative Commons Attribution (CC BY) license (http://creativecommons.org/licenses/by/4.0/). 